\title{
Influenza dell' aria rarefatta sull' ontogenesi.
}

Nota I. La perspirazione delle ova di gallina durante lo sviluppo in alta montagna.

Per

Dott. Alberto Aggazzotti, Libero docente di fisiologia.

(Laboratori scientifici $*$ A. Mosso * sul Monte Rosa e Istituto fisiologico di Torino.)

Con 1 figura nel testo e 4 tavole.

Eingegangen am 27. Januar 1913.

Chi ha fatto ascensioni in alta montagna avrà certamente osservato quanto intensa sia talora la sete, e quanto grande e molesto diventi il senso di secchezza alle fauci. - Nelle giornate serene e molto fredde, quando l'umidità è minima e il vento forte, le mucose del naso e delle labbra spesso screpolano e sanguinano, perchè l'eraporazione alla superficie delle mucose e della pelle è rapida e intensa. Se la marcia è faticosa e la temperatura non molto bassa si può osservare che anche l'abbondante sudore asciuga con grande rapidità e facilità sulla pelle, e che non raramente si forma nei punti di massima sudorazione un evidente deposito di cloruro sodico. Questi fatti erano facilmente spiegabili per le speciali condizioni fisiche del clima in alta montagna, la diminuita pressione barometrica e la secchezza dell' aria, che aumentano l'evaporazione dell' acqua.

Anche la perdita fortissima in peso, che si osserva talora nell'uomo dopo un'ascensione in montagna, perdita che può essere di $5-6 \mathrm{Kg}$. in una marcia di $6-7$ ore, sopra i 3000 metri, si può facilmente interpretare colla maggior perdita di acqua a grandi altezze. Non solo nelle marcie, ma anche nel riposo parve che per le speciali condizioni del clima alpino, dovesse avrenire in alta montagna una disidratazione dell'organismo: e per deduzione si ammise 
(GRAwITz) ${ }^{1}$ ) che la più attiva perdita di acqua in montagna provocasse una maggior concentrazione del sangue e indirettamente fosse la causa dell' iperglobulia alpina.

REGNARD $\left.{ }^{2}\right)$ per mezzo di un suo apparecchio mantenne lungamente animali sotto alla campana pneumatica e determinò il vapor d'acqua ehe essi eliminavano; egli credette di aver dimostrata questa disidratazione, avendo trovato che nell' aria anche lievemente rarefatta gli animali emettono una maggior quantità di acqua.

$1^{\mathrm{a}}$ Tortora di $140 \mathrm{gr}$.

a $760 \mathrm{~mm}$. elimina gr. 5.04 d'acqua,

a 500 - $\quad-\quad 9.12 \quad$ -

2 ' Tortora di $130 \mathrm{gr}$.

a $756 \mathrm{~mm}$. elimina gr. 4.31 d'acqua,

a $500 \quad-\quad$ - 6.72

L'esperienza di REgNaRd non ha avuto conferma, come non siè dimostrata vera l'ipotesi della maggior concentrazione del sangue in montagna.

Le esperienze, che sono state fatte più recentemente sotto alla campana pneumatica e direttamente nei Laboratorii a grande altezza, dimostrano che la perdita complessiva dell'acqua nell'aria rarefatta, durante il riposo, nell'nomo e negli animali, è minore che alla pressione normale. SchrötTER e ZUNTZ ${ }^{3}$ ), in due ascensioni aereostatiche trovarono che la quantità di vapor d'acqua espirâto a grandi altezze è minore che al piano. C. Foít) ha veduto su se stesso che sotto alla campana pneumatica, ad una pressione di $460 \mathrm{~mm}$., nello spazio di 1 ora, si perde in peso meno che nell'eguale spazio di tempo alla pressione normale. Alla pressione di Torino, $740 \mathrm{~mm}$., egli trova in due esperienze una perdita di peso di 60 e 66 gr.; sotto alla campana pneumatica solo 48 e $38 \mathrm{gr}$.

A. Mosso ${ }^{5}$ ) nella seconda spedizione scientifica fatta sul Monte

1) E. Grawitz, Über die Einwirkung des Höhenklimas auf die Zusammensetzung des Blutes. Berl. klin. Wochenschr. Nr. 33 e seg. 1895.

2) Regnard, Cure d'altitude. 1897. Cit. Traité de Physique Biologique. T. 1. p. 1094.

3) H. SCHRötter a. N. Zuntz, Ergebnisse zweier Ballonfahrten zu physiologischen Zwecken. Pflügers Arch. Bd. 92 . S. 479.

4) C. Fò̀, Critica sperimentale delle ipotesi emesse per spiegare l'hyperglubulia dell' alta montagna. Rend. R. Acc. dei Lincei. Vol. 12. Fasc. 10. 1903. e Laboratoire scientifique international du Mont Rosa. Vol. 1. Torino 1904.

5) A. Mosso, L'uomo sulle Alpi. III. ediz. Treves Milano 1909. p. 393. 
Rosa nel 1903 ha studiato la perdita in peso dell'uomo alla Capanna Regina Margherita (4560 m.), comparativamente a quella che si ha a Torino; le determinazioni venivano fatte pesando direttamente un uomo vestito con una stadera sensibile ai 4 grammi. Egli trovò che la perspirazione è minore sulle Alpi e ne attribuì la causa alla bassa temperatura, che facendo restringere $i$ vasi sanguigni determina una circolazione cutanea meno attiva e una minor sudorazione; in alcuni casi la perdita in peso sulle Alpi era metà che a Torino. 亡́ da escludersi che una minore eliminazione di anidride carbonica potesse mascherare una più forte emissione di vapori d'acqua, perché numerose esperienze tendono a dimostrare piuttosto il contrario.

Zuntz, Loewy, Müller e Caspari ${ }^{1}$, pure sul Monte Rosa, osservarono che il corpo umano complessivamente perde meno acqua che al piano, perchè la bassa temperatura toglie ogni attività alle ghiandole sudorifere.

H. Guillemard e R. Moog ${ }^{2}$ ) hanno stadiato l'influenza dell'aria rarefatta sull' eliminazione dell' acqua nelle cavie. Essi sottoposero gli animali ad pna pressione di $370 \mathrm{~mm}$. (5.835 m.) per 20 ore, e ottennero questi risultati: acqua perduta in 24 ore a $756 \mathrm{~mm}$. gr. 12.08 , a $387 \mathrm{~mm}$. gr. 10.65 . - In altre esperienze ${ }^{3}$ ) dove riprodussero meglio sotto alla campana pneumatica le condizioni che si hanno sull'alta montagna (pressione 420 , temp. $5-10^{\circ} \mathrm{C}$., aria secca) trovarono in tre cavie un'eliminazione media di gr. 11.41, mentre che riproducendo le condizioni che si hanno al livello del mare (pressione 760 , temp. $30-35^{\circ}$ C., aria satura d'acqua a $10^{\circ} \mathrm{C}$.) trovarono in media una eliminazione di gr. 23.79. Gli stessi autori ${ }^{4}$ ) hanno studiato anche su loro stessi l'influenza che il clima dell'alta montagna ha sulla disidratazione dell'organismo, determinando separatamente la perdita d'acqua per i polmoni e per la pelle al piano e in montagna. Il vapor d'acqua emesso dai polmoni veniva trattenuto con $\mathrm{CaCl}_{2}$ anidro e $\mathrm{H}_{2} \mathrm{SO}_{4}$, e determinato per pesata. - L'esperimento durava $1 / 2$ ora. - Il vapor d'acqua emesso dalla pelle veniva determinato pure per pesata, ma l'esperimento si faceva su una regione

1) Zuntz, Lofwy, Müller, CASPaRI, Höhenklima und Bergwanderungen. Deutsehes Verlagshaus Bong u. Co. Berlin 1906. S. 378.

2) H. Guillemard et R. Moog, Recherches expérimentales sur l'exalation de vapeurs d'eau. Compt. Rend. Soc. Biol. T. 1. 1907. p. 819.

3) - Compt. Rend. Soc. Biol. T. 1. 1907. p. 874.

4) - - Influence du climat d'altitude sur la déshydratation de l'organisme. Compt. Rend. Acc. de Scien. T. 145. 1907. p. 823. 
molto limitata; essi mantenevano il palmo della mano per $1 / 4$ di ora applicato sopra l'orlo di un cristallizzatore di $4.5 \mathrm{~cm}$. di diametro con $\mathrm{H}_{2} \mathrm{SO}_{4}$. Guillemard e Moog trovarono che l'acqua emessa complessivamente dai polmoni, nell'unità di tempo, non cambia sensibilmente coll'altezza, ma poichè diminuisce progressivamente quella contenuta nell'aria inspirata, in realtà, la quantità di acqua emessa dai polmoni cresce sensibilmente in alta montagna. La perdita d'acqua per la pelle invece diminuisce molto in alta montagna e poichè essi ammettono che essa è 3--4 volte, almeno, maggiore dell' acqua perduta per i polmoni, così concludono che l'organismo nell'aria rarefatta s'arricchisce di acqua e aumenta di peso. I valori ottenuti nelle loro esperienze sono $i$ seguenti:

\begin{tabular}{|c|c|c|c|c|}
\hline & Parigi & $\begin{array}{l}\text { Chamonix } \\
1050 \mathrm{~m}\end{array}$ & $\begin{array}{l}\text { Grands-Mulets } \\
3050 \mathrm{~m} .\end{array}$ & $\begin{array}{l}\text { Iront-Blane } \\
4810 \mathrm{~m} \text {. }\end{array}$ \\
\hline $\begin{array}{l}\text { Acqua eliminata coll aria } \\
\text { espirata, al } \mathrm{m}^{\text {to }} \cdots\end{array}$ & $0.275 \check{7} \mathrm{gr}$. & $0.2669 \mathrm{gr}$. & $0.2511 \mathrm{gr}$. & $0.2738 \mathrm{gr}$. \\
\hline $\begin{array}{l}\text { Acqua eliminata dai pol- } \\
\text { moni, al mto }\end{array}$ & 0.1826 & $0.1781-$ & $0.2009-$ & 0.2418 \\
\hline $\begin{array}{c}\text { Acqua eliminata per la } \\
\text { pelle, al mto } . . .\end{array}$ & $0.043-$ & 0.026 & 0.016 & 0.010 \\
\hline
\end{tabular}

0. Cohnhem ${ }^{1,2}$ ) e i suoi collaboratori hanno fatto in due spedizioni scientifiche sul Monte Rosa numerose esperienze sull'uomo per determinare la perdita in peso complessiva durante la notte e sono arrivati alla conclusione che in alta montagna v'è una maggior perdita di peso che al piano, ciò che essi attribuiscono ad una maggior eliminazione di acqua. Le medie delle perdite in peso sono le seguenti:

\begin{tabular}{|c|c|c|c|c|}
\hline & Connhem & KREGLINGER & TOBLER & WEBER \\
\hline $\begin{array}{l}\text { Al Piano . . . . . } \\
\text { Al Colle d'Olen, } 2900 \mathrm{~m} . \\
\text { Alla Capanna Margherita, } \\
4560 \mathrm{~m} . \quad \text {. . . . . . }\end{array}$ & $\begin{array}{l}287 \mathrm{gr} . \\
334= \\
300=\end{array}$ & $\begin{array}{l}256 \mathrm{gr} . \\
516= \\
375=\end{array}$ & $\begin{array}{l}225 \mathrm{gr} . \\
450- \\
333-\end{array}$ & $\begin{array}{l}-\overline{\mathrm{gr}} \\
350 \\
325-\end{array}$ \\
\hline
\end{tabular}

1) 0. Cohnheim, G. Kreglinger u. Kreglinger (Sohn), Beiträge zar Physiologie des Wassers und des Kochsalzes. Zeitschr. f. physiol. Chem. Bd. 63. S. 413 e Atti dei Labor. scient. 'A. Mosso sul Monte Rosa. Vol. 3. p. 22.

2) 0. Cohnheim, G. Kreglinger, L. Tobler, 0. H. Weber, Die Physiologie des Wassers und des Kochsalzes. Zeitschr. f. physiol. Chem. Bd. 78. S. 62 e Atti dei Labor. scient. >A. Mosso sul Monte Rosa. Vol. 4. 
Come si vede da questi valori, la maggior perdita di peso si sarebbe solo verificata negli individui quando passarono dal piano al Col d'Olen, e non quando passarono dall'Olen alla Capanna Regina Margherita, sebbene il dislivello in questo secondo caso fosse pure forte, $1600 \mathrm{~m}$. Perciò, anche queste ricerche di Connerem solo in parte non concordano con quelle fatte da tutti gli altri studiosi sullo stesso argomento.

Recentissimamente il Prof. Galeottr col suo assistente Dott. SignoRELLI hanno fatto nei Laboratori scientifici del Monte Rosa nuove ricerche sul bilancio dell' acqua, nel riposo e nella fatica e sono arrivati anch'essi alla conclusione che in alta montagna diminuisce la perdita dell'acqua. In queste esperienze gli Autori hanno studiato il ricambio dell'acqua più completamente di quanto fecero i ricercatori precedenti; essi non solo determinarono l'acqua emessa colle orine, colle feci, coll'aria respirata, e, indirettamente, quella emessa dalla pelle, ma tennero calcolo anche dell'aequa introdotta colle bevande, con gli alimenti solidi, e di quella formatasi per le ossidazioni intraorganiche. Le medie ottenute nelle loro esperienze sul ricambio dell' acqua nel riposo si possono riunire nelle seguenti due tavole, in cui $i$ valori sono espressi in ettogrammi:

\begin{tabular}{|c|c|c|c|c|}
\hline & \multicolumn{2}{|c|}{ GaLeotti } & \multicolumn{2}{|c|}{ SigNORELLI } \\
\hline & Acqua intr. & Aequa emessa & Aequa intr. & Acqua emessa \\
\hline Napoli, $0 \mathrm{~m} . . .$. & 27.9 & 31.0 & 21.5 & 27.0 \\
\hline Colle d'Olen, $2900 \mathrm{~m}$. . . & 30.1 & 25.7 & - & - \\
\hline Capan. Margherita, $4560 \mathrm{~m}$. & - & - & 24.7 & 23.6 \\
\hline
\end{tabular}

\begin{tabular}{|c|c|c|c|c|c|c|}
\hline & \multicolumn{3}{|c|}{$\begin{array}{l}\text { GALEOTTI } \\
\text { acqua emessa }\end{array}$} & \multicolumn{3}{|c|}{$\begin{array}{l}\text { SIGNORELLI } \\
\text { acqua emessa }\end{array}$} \\
\hline & $\begin{array}{c}\text { colle feci } \\
\text { o coll' orina }\end{array}$ & $\begin{array}{c}\text { coll' aria } \\
\text { espirata }\end{array}$ & $\begin{array}{c}\text { coll' evaporaz. } \\
\text { cutanea }\end{array}$ & $\begin{array}{c}\text { colle feci } \\
\text { e coll orina }\end{array}$ & $\begin{array}{l}\text { coll' aria } \\
\text { espirata }\end{array}$ & $\mid \begin{array}{c}\text { coll' eraporaz. } \\
\text { entanea }\end{array}$ \\
\hline Napoli. . . & 14.52 & 3.48 & 13.05 & 13.94 & 4.61 & 8.50 \\
\hline Colle d'Olen. . & 16.02 & 3.37 & 6.47 & - & 一 & - \\
\hline Cap. Margherita & - & - & - & 13.50 & 4.68 & 6.47 \\
\hline
\end{tabular}

I valori ottenuti da Galeotti e Signorelli dimostrano che in alta montagna il ricambio dell'acqua è molto diverso che al piano: non solo l'acqua emessa in montagna è minore in quantità assoluta, ma essa è anche minore rispetto a quella introdotta. La diminuzione maggiore si osserva per l'acqua emessa dalla pelle; per quella eliminata dai reni, dall'intestino e dai polmoni non si osserva grande dif- 
ferenza in montagna e al piano. Ciò è contrario a quanto avevano ammesso Connheim e i suoi collaboratori ${ }^{1}$, che cioè l'eliminazione dell'acqua per la pelle sentisse poco o punto l'influenza dell'alta montagna, ma che questa agisse specialmente sull'eliminazione dell'acqua pei polmoni. Nell'uomo, essi dicevano, la maggior parte dell'aequa si elimina col sudore, ma solo quando entrano in funzione le ghiandole sudorifere per stimoli a loro specifici e indipendenti dalla pressione e dal clima alpino; se non vi sono questi stimoli, non si elimina acqua per la pelle. Diversamente avverebbe per l'acqua eliminata per i polmoni; l'aria che arriva in contatto colle mucose si riscalda alla temperatura del corpo e si carica di acqua, ma poichè in montagna l'aria è sempre più secca e più fredda e l'evaporazione è più facile, la quantità di acqua eliminata dai polmoni deve essere maggiore in montagna, che al piano. Forse la causa della divergenza sta in ciò, che CoHsherm determinò la perdita di peso durante la notte e GaLEortr anche durante il giorno. Nella notte, sotto le coltri del letto, aleune condizioni fisiche (temperatura e igroscopia), sono diverse che nel giorno.

Noi possiamo adunque ritenere che, sebbene molte condizioni fisiche del clima alpino sieno favorevoli ad una maggior perdita di acqua, tuttavia, forse per una speciale reazione del corpo alle nuove condizioni dell'ambiente, e, in specie, alla bassa temperatura, la quantità di acqua complessivamente emessa è minore in montagna che al piano.

Le esperienze sul bilancio dell' acqua nell' uomo in montagna sono difficili, perchè non sempre si può tenere una dieta rigorosamente costante e non si può conoscere con esattezza la quantità di acqua introdotta colle bevande e coi cibi, inoltre l'eliminazione dell'acqua si fa per varie vie, per i reni, per lintestino, per i polmoni e per la pelle. Avendo in corso da vari anni esperienze sullo sviluppo delle ova nell'aria rarefatta, ho voluto studiare anche il ricambio dell'acqua, e ho seguito, con una bilancia sensibile al centigrammo, passo passo la perdita di peso delle ova, durante l'incubazione al piano e in montagna.

L'acqua che si forma nell'organismo umano per ossidazione dei nutrimenti ingeriti non può sempre essere calcolata, variando essa non solo secondo la quantità, ma anche secondo la qualità degli alimenti: l'errore può essere specialmente forte nel calcolo dell' acqua

1) loc. cit. p. 414 . 
prodottasi nell' ossidazione delle sostanze proteiche e dei grassi ingeriti, giacchè essi non arrivano sempre al loro massimo di ossidazione, e possono dare prodotti intermedi più o meno ossidati: ma nell'uovo in via di sviluppo le sostanze nutritive, che vanno gradatamente ossidandosi e trasformandosi, si possono ritenere sempre costanti, e l'intensità dei processi ossidativi, nelle diverse ova pure costante. Inoltre, pel fatto noto che la perdita in peso dell'ovo in via di sviluppo è pressapoco simile a quella dell'ovo non fecondato durante l'incubazione, noi possiamo dedurre che la perdita in peso dell'ovo durante lineubazione non dipende dall'intensità maggiore o minore dei processi ossidativi interni dell'ovo, e nemmeno dal ricambio respiratorio del pulcino in ria di sviluppo. Dalle esperienze di HaSSELbalch ${ }^{1}$ ) sappiamo che, nei primi tre giorni di incubazione, il ricambio respiratorio dell'ovo fecondato è molto ịrregolare, perchè v'è emissione di $\mathrm{O}_{2}$ e $\mathrm{CO}_{2}$ e contemporaneamente assorbimento di $\mathrm{O}_{2}$. Trascorso il primo giorno di incubazione, il consumo di ossigeno ha il sopravento, al terzo giorno l'assorbimento dell'ossigeno e l'eliminazione dell'anidride carbonica hanno raggiunto valori che in peso pressapoco si corrispondono. Infatti il Q. R. è in media $0.677(0.606-0.734)$, perciò dopo il $3^{\circ}$ giorno il ricambio respiratorio non produce importanti cambiamenti nel peso dell'ovo. - Una perfetta corrispondenza in peso fra $\mathrm{l}^{\prime} \mathrm{O}_{2}$ e il $\mathrm{CO}_{2}$ si ha quando il Q. R. è-0.727. Solo quando lo sviluppo del pulcino è molto avanzato, dopo il $17^{\circ}$ giorno, secondo PotT e Prever ${ }^{2}$ ) il $\mathrm{CO}_{2}$ eliminato aumenta in proporzione più dell' $\mathrm{O}_{2}$ assorbito e il peso dell'ovo diminuisce; tuttavia, come ho detto, la perdita complessiva di peso nell'ovo fecondato e non fecondato dopo 20 giorni di incubazione è pressapoco uguale, ed anche nelle esperienze descritte da Pott e Preyer non si nota una diminuzione del peso più evidente nelle ova in via di sviluppo.

Nelle nostre esperienze studieremo l'influenza che hanno sulla perdita in peso delle ova solo alcune condizioni del clima alpino: la diminuita pressione barometrica e la conseguente più bassa tensione del rapore d'acqua nell'aria ambiente, mentre rimangono costanti la temperatura, l'umidità relativa e tutte le altre condizioni d'esperimento, indispensabili per un regolare sviluppo delle ova al piano. Abbiamo

1) K. A. Hasselbalch, Über die respiratorischen Stoffwechsel des Hühnerembryos. Skandin. Arch. Bd. 10. 1900. S. 353.

2) R. Potr u. W. Preyer, Über den Gaswechsel und die chemischen Veränderangen des Hühnereies während der Bebrütung. PfLÜGers Arch. Bd. 27. 1882. S. 35̃2. 
veduto che il clima alpino diminuisce l'eliminazione dell'acqua nell'nomo, e nell'animale adulto in riposo; ora vedremo che l'aria rarefatta agisce sull'ovo in via di sviluppo in modo diverso. È noto che il pulcino durante il suo sviluppo si comporta come un animale poichilotermo (PEMBREY 1), il suo ricambio respiratorio aumenta coll'aumentare della temperatura e viceversa; solo dopo la nascita esso acquista i poteri regolatori che hanno gli animali adulti a sangue caldo, in essi il $\mathrm{CO}_{2}$ eliminato diminuisce aumentando la temperatura. Verso la fine dell'incubazione, v'è un periodo di passaggio in cui la temperatura non ha un' azione costante.

Per quanto mi consta nessuno aveva sino ad oggi studiato linfluenza dell' aria rarefatta sullo sviluppo ontogenetico delle ova; forse, per la difficoltà di avere una regolare incubazione sotto alla campana pneumatica 0 in alta montagna. Snlle Alpi oltre i $2500 \mathrm{~m}$. è stato tentato da qualche albergatore un allevamento di galline, ma, dalle informazioni raccolte, pare che le galline cessassero di fare ova quando venivano trasportate in alto e interromperano l'incubazione prima del tempo se l'averano incominciata.

Nei Laboratorii Scientifici A. Mosso* sul Monte Rosa ho potato comodamente intraprendere ricerche sullo sviluppo delle ova; in questa prima Nota mi limiterò di riferire i risultati sulla loro perdita in peso.

Prout ${ }^{2}$ ), Voit ${ }^{3}$ ), Baumgärtner ${ }^{4}$ ) Baudrimont e Martin St. Anges 5), Pott e Preyer ${ }^{6}$ ) hanno studiato la perdita in peso delle ora fecondate e non fecondate durante l'incubazione al piano. I valori ottenuti da questi ricercatori non sono concordanti fra loro, perchè l'incubazione non avpeniva sempre nelle stesse condizioni, e non ci possono servire come termine di confronto colle nostre esperienze in alta montagna.

Per poter meglio apprezzare l'influenza dell'aria rarefatta salla perdita in peso delle ova ho fatto esperienze comparative a Torino, nel Laboratorio di Fisiologia (250 m. s. m.) ed al Colle d'Olen, nel

1) M. S. Pembrex, On the reponse of the chick before and after hatching to changes of external temperature. Journ. of Physiol. Vol. 17. p. 331 e Maly. Tol. 25. p. 401.

2) Prout, Philosoph. Transact. Roy. Soc. London. Vol. II. 1822. p. 377.

3) C. Vort, Über das Verhalten der Kalkschalen der Hühnereier bei der Bebrititang. Zeitschr. f. Biologie. Bd. 13. 1877. S. 518.

4) J. Baumgärtner, Der Athmungaprozeß in Ei. Freiburg i. B. 1861.

5) Baudrimont et Martin St. Anges, Annales de chimio et de physique. Troisième série. Vol. 21. p. 205. Paris 1847.

6) R. Pott u. W. Preyer, loc. cit. S. 323. 
Tavola I. Colle d'Olen.

4 Agosto 1911.

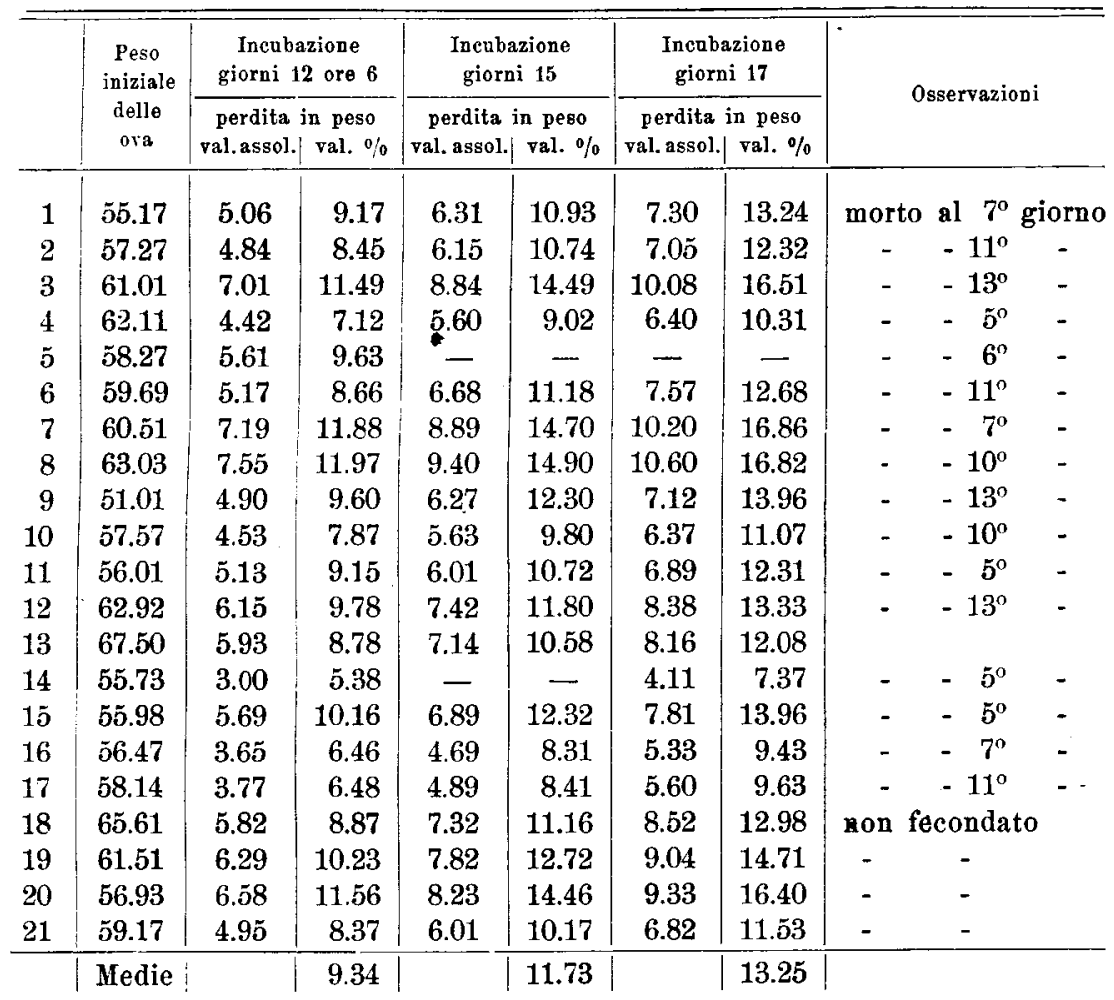

Laboratorio scientifico ,A. Mosso (2900 m. s. m.). Le esperienze vennero compiute al Colle d'Olen con la stessa incubatrice (sistema Hearcon) che aveva adoperata a Torino, per mantenermi nelle identiche condizioni d'esperimento. L'incubazione venne sempre fatta a $39^{\circ} \tilde{5}-40^{\circ}$ : e si osservarono strettamente tutte le regole richieste per un allevamento razionale delle ova: a Torino, infatti, lo sviluppo ba sempre progredito regolarmente, la percentuale delle nascite era molto alta $(80 \%)$. Se al Colle d'Olen non si ottenne un completo sviluppo delle ova e gli embrioni morirono entro la seconda settimana, non si deve per ciò incolpare la tecnica usata nel fare l'incubazione, che era sempre la stessa a Torino e al Colle d'Olen, ma altre cause e forse l'azione esercitata dalla diminuita pressione.

Le uova per gli esperimenti di Torino venirano prese fresche in un pollaio vicino alla città, prima di metterle nell'incubatrice erano lavate in acqua tiepida, asciugate e lasciate riposare per 24 ore alla 
Tavola II. Colle d'Olen.

2õ Agosto 1911.

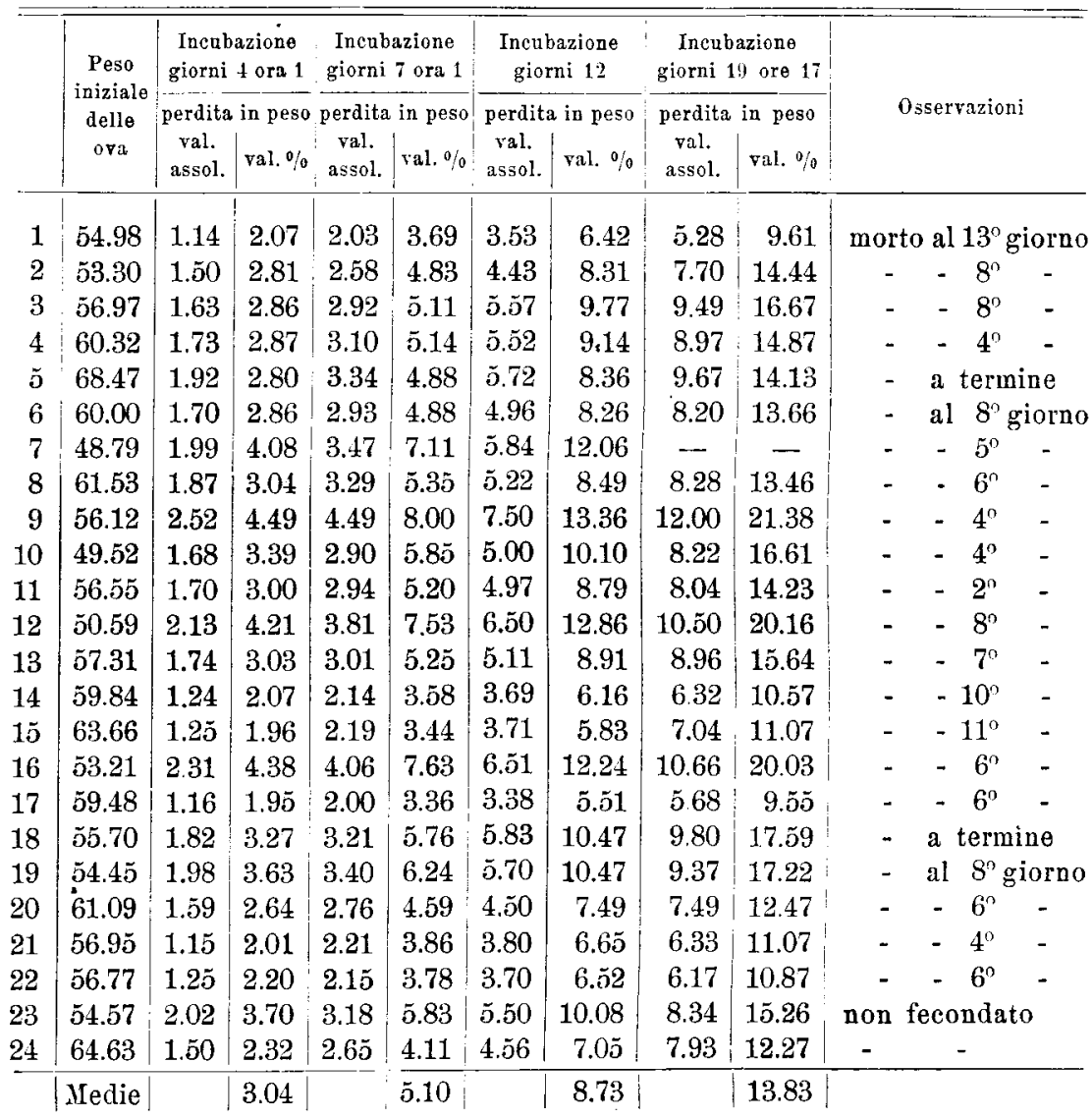

temperatura ambiente $\left(20^{\circ}\right.$. Le ova per la esperienze sul Monte Rosa, venivano prese a Torino o a Modena e per mezzo di pacchi postali spedite ad Alagna $(150 \mathrm{Km}$. di ferrovia) e di qui trasportate subito ai Laboratorii del Colle d'Olen (4 ore di mulo); esse impiegavano in media sei giorni per arrivare a destinazione. Come controllo metà delle ova venivano nuovamente spedita al piano per essere incubate. Anche al Colle d'Olen le ova venivano messe nell'incubatrice solo dopo un riposo di 24 ore alla temperatura ambiente $\left(8^{\circ}\right)$ e dopo essere state lavate nell'acqua tiepida. Durante tutto il periodo di incubazione non si lasciò mai venir meno l'acqua nel serbatoio apposito dell'incubatrice, per mantenere l'aria calda satura di vapori. Le ova renivano ogni giorno rivoltate e, nel secondo periodo del- 
Tavola III. Colle d'Olen.

26 Agosto 1912.

\begin{tabular}{|c|c|c|c|c|c|}
\hline & \multirow{2}{*}{$\begin{array}{l}\text { Peso iniziale } \\
\text { delle ova }\end{array}$} & \multicolumn{2}{|c|}{$\begin{array}{l}\text { Incubazione } \\
\text { giorni } 5 \text { ora } 1\end{array}$} & \multicolumn{2}{|c|}{$\begin{array}{l}\text { Incubazione } \\
\text { giorni } 9 \text { ore } 12\end{array}$} \\
\hline & & \multicolumn{2}{|c|}{ perdita in peso } & \multicolumn{2}{|c|}{ perdita in peso } \\
\hline 1 & 73.70 & 3.55 & 4.81 & อ..34 & 7.24 \\
\hline 2 & 54.62 & 3.17 & 5.80 & 5.03 & 9.22 \\
\hline 3 & 62.23 & 3.18 & 5.11 & 4.97 & 8.00 \\
\hline 4 & 74.80 & 2.30 & 3.08 & 3.81 & 5.10 \\
\hline 5 & 60.84 & 2.06 & 3.58 & 3.23 & 5.30 \\
\hline 6 & 59.42 & 1.87 & 3.14 & 3.03 & 5.10 \\
\hline 7 & 43.12 & 1.87 & 4.33 & 3.02 & 7.01 \\
\hline 8 & 47.60 & $1.8 \tilde{5}$ & 3.88 & 2.76 & 5.80 \\
\hline 9 & 59.23 & 2.95 & 4.98 & 4.40 & 7.43 \\
\hline 10 & 61.54 & 3.94 & 6.40 & 6.20 & 10.09 \\
\hline 11 & 55.12 & 4.54 & 8.24 & 7.42 & 13.30 \\
\hline 12 & 56.56 & 2.86 & 4.80 & 4.26 & 7.15 \\
\hline 13 & 67.91 & 2.56 & 3.77 & 3.90 & 5.75 \\
\hline 14 & 54.74 & 3.04 & $\tilde{\boldsymbol{5} . \tilde{5} \tilde{5}}$ & 5.00 & 9.12 \\
\hline $1 \check{0}$ & $54.1 \check{0}$ & 1.87 & 3.45 & 2.98 & 5.50 \\
\hline 16 & $49.8 \tilde{5}$ & 2.15 & 4.31 & 3.55 & 7.11 \\
\hline 17 & 60.80 & 2.30 & 3.78 & 3.53 & 5.80 \\
\hline 18 & 55.25 & 2.30 & 4.16 & 3.89 & 7.05 \\
\hline 19 & 61.65 & 3.00 & 4.86 & 4.57 & 7.40 \\
\hline 20 & 55.95 & 2.77 & 4.95 & 4.18 & 7.47 \\
\hline 21 & $56.6 \check{~}$ & 2.95 & 5.20 & 4.59 & 8.10 \\
\hline \multirow[t]{2}{*}{22} & 59.51 & 3.51 & 5.90 & 5.53 & 9.31 \\
\hline & Medie & & 4.59 & & 7.42 \\
\hline
\end{tabular}

l'incubazione, esposte momentaneamente all'aria, secondo le regole note: al Colle d'Olen, essendo la temperatura ambiente assai bassa, ebbi cura che l'esposizione all' aria fosse più breve. Le pesate si facevano ogni $4-5$ giorni e il più rapidamente possibile; per impedire un troppo forte e rapido raffreddamento delle ova, queste erano pesate entro una scatola e coperte di ovatta. La bilancia a giogo era sensibile al centigrammo. Nelle Tavole seguenti sono riuniti i singoli gruppi di esperienze: nella prima colonna è segnato il peso delle ova in gr., prima di incominciare l'incubazione; nelle colonne successive sono registrate le perdite in peso in valori assoluti $e$ in proporzione a $100 \mathrm{gr}$., per ogni diverso periodo di incubazione. Nell'ultima colonna sono segnate le osservazioni su ciascun ovo. Alla fine dell'esperimento le ova non nate venivano rotte e, se contenevano un embrione morto, se ne valutava l'età dalla sua lunghezza. Le ova 
Tavola IV. Torino.

20 Aprile 1912.

\begin{tabular}{|c|c|c|c|c|c|c|c|c|c|c|}
\hline \multirow{2}{*}{\multicolumn{2}{|c|}{$\begin{array}{c}\text { Peso } \\
\text { iniziale } \\
\text { delle ova }\end{array}$}} & \multicolumn{2}{|c|}{$\begin{array}{c}\text { Incubazione } \\
\text { giorni } 4 \text { ore } 3\end{array}$} & \multicolumn{2}{|c|}{$\begin{array}{c}\text { Incubazione } \\
\text { giorni } 9 \text { ore } 3\end{array}$} & \multicolumn{2}{|c|}{$\begin{array}{c}\text { Inenbazione } \\
\text { giorni } 15 \text { ore } 3\end{array}$} & \multicolumn{2}{|c|}{$\begin{array}{l}\text { Incubazione } \\
\text { giomi } 21 \text { ora } 1\end{array}$} & \multirow{2}{*}{ Osservazioni } \\
\hline & & \multicolumn{2}{|c|}{$\begin{array}{c}\text { perdita in peso } \\
\text { ral. assol.| val. } \%\end{array}$} & \multicolumn{2}{|c|}{$\begin{array}{l}\text { perdita in peso } \\
\text { val. assol.| ral. } \%\end{array}$} & \multicolumn{2}{|c|}{\begin{tabular}{|} 
perdita in peso \\
val. assol. val. $\%$
\end{tabular}} & \multicolumn{2}{|c|}{$\begin{array}{c}\text { perdita in peso } \\
\text { val. assol. | val. } \%\end{array}$} & \\
\hline 1 & 58.64 & $1 . \check{0} 0$ & 2.56 & 3.37 & 5.57 & 5.87 & 10.06 & - & - & nato \\
\hline 2 & 63.77 & 1.83 & 2.87 & 3.85 & 6.04 & 6.43 & 10.10 & 8.52 & 13.37 & \multirow{4}{*}{$\begin{array}{l}\text { morto a termine } \\
\text { nato } \\
\text { rotto al } 15^{\circ} \text { giorno } \\
\text { nato }\end{array}$} \\
\hline 3 & 53.69 & 1.32 & 2.45 & 2.90 & 5.39 & 5.10 & 9.50 & - & - & \\
\hline 4 & 56.92 & 1.65 & 2.90 & 3.63 & 6.38 & 6.06 & 10.65 & - & $\longrightarrow$ & \\
\hline 5 & 54.04 & 1.18 & 2.18 & 2.52 & 4.66 & 4.52 & 8.36 & - & - & \\
\hline 6 & 61.02 & 1.68 & 2.75 & 3.73 & 6.11 & 6.50 & 10.64 & - & - & - \\
\hline 7 & 54.40 & 1.51 & 2.77 & 3.38 & 6.21 & 5.70 & 10.47 & - & - & \\
\hline 8 & $\tilde{0} 4.87$ & 1.35 & 2.46 & 2.90 & 5.28 & 5.15 & 9.38 & - & - & \\
\hline 9 & 57.35 & - & - & 2.58 & 4.50 & 6.13 & 10.69 & 8.30 & 14.48 & \multirow{3}{*}{$\begin{array}{l}\text { morto al } 6^{\circ} \text { giorno } \\
\text { rotto al } 17^{\circ} \text { giornc } \\
\text { nato }\end{array}$} \\
\hline 10 & 50.74 & 1.55 & 3.05 & 3.60 & 7.09 & 6.24 & 12.29 & - & - & \\
\hline 11 & 54.20 & 1.50 & 2.76 & 3.40 & 6.27 & 6.08 & 11.23 & - & 一 & \\
\hline 12 & 60.37 & 1.65 & 2.73 & 3.65 & 6.05 & 6.33 & 10.48 & - & - & \multirow{3}{*}{$\begin{array}{l}\text { - } \\
\text { morto a termine } \\
\text { rotto al } 9^{\circ} \text { giorno }\end{array}$} \\
\hline 13 & 53.89 & 1.27 & 2.35 & 2.82 & 5.23 & 5.02 & 9.31 & 7.21 & 13.39 & \\
\hline 14 & 49.43 & 1.36 & 275 & 3.02 & 6.11 & - & - & - & - & \\
\hline 15 & 53.84 & 1.14 & 2.12 & - & - & - & 一 & - & - & $-\quad-5^{0}-$ \\
\hline 16 & 55.52 & 1.54 & 2.77 & - & - & - & - & - & - & $-\quad-5^{\circ}$ \\
\hline 17 & 59.30 & 1.78 & 3.00 & 3.90 & 6.57 & - & - & - & - & $-9^{2}$ \\
\hline 18 & 54.00 & 1.29 & 2.38 & - & - & - & - & - & - & $-5^{n}$ \\
\hline 19 & 53.02 & 1.44 & 2.71 & 3.21 & 6.05 & 5.83 & 11.00 & - & - & nato \\
\hline 20 & 50.62 & 1.30 & 2.56 & 2.90 & 5.72 & 5.28 & 10.43 & - & - & - \\
\hline 21 & 57.72 & 1.24 & 2.14 & 2.77 & 4.80 & 4.77 & 8.26 & - & - & - \\
\hline 22 & 52.80 & 1.15 & 2.17 & 2.60 & 4.92 & 4.56 & 8.61 & - & 一 & - \\
\hline 23 & 65.52 & 1.98 & 3.02 & 4.35 & 6.62 & 7.68 & 11.73 & 10.94 & 16.71 & non fecondato \\
\hline 24 & 50.74 & 1.31 & 2.58 & 3.40 & 6.70 & - & - & - & - & $-\quad \quad-$ \\
\hline 25 & 5̆7.47 & 1.35 & 2.35 & 3.52 & 6.12 & 6.03 & 10.49 & 8.73 & 15.19 & - \\
\hline 26 & 61.04 & 1.33 & 2.17 & 3.46 & 5.66 . & 5.99 & 9.81 & - & - & - \\
\hline 27 & 57.15 & 1.17 & 2.04 & 2.63 & 4.60 & 4.38 & 7.66 & $\ldots$ & - & - \\
\hline 28 & 49.40 & 1.28 & 2.59 & 2.80 & 5.67 & 4.96 & 10.04 & 7.03 & 14.24 & - \\
\hline 29 & 53.91 & 1.36 & 2.52 & 2.94 & 5.45 & 5.19 & 9.62 & - & - & - \\
\hline 30 & 60.81 & 1.84 & 3.02 & 4.12 & 6.77 & 6.40 & 10.53 & - & - & - \\
\hline & Medie & & 2.56 & & 5.83 & & 10.03 & & 14.55 & \\
\hline
\end{tabular}

che non presentavano traccia di sviłuppo, venivano classificate come ova non fecondate; le perdite in peso subite da queste ultime ova sono registrate in fondo ad ogni tavola.

L'esperimento III incominciato il 26 agosto fu interrotto al 4 Settembre, perchè, causa il cattivo tempo, fummo costretti a chiudere innanzi tempo i Laboratori; l'ultima pesata venne fatta dopo 9 giorni e 12 ore di incubazione, le ova non vennero esaminate internamente alla fine dell'esperimento. 
Nell'esperimento IV, fatto a Torino, di alcune ova si hanno soltanto le prime pesate, perchè contemporaneamente alle esperienze sulla perdita in peso, si studiava anche la reazione dei liquidi dell'ovo durante lo sviluppo e per queste ultime esperienze si dovevano rompere le ova a diversi periodi di incubazione.

Nelle tre esperienze fatte al Colle d'Olen le ova durante l'incubazione hanno avuto una perdita in peso maggiore di quella presentata al piano: la differenza è abbastanza forte ed è costante. Per potere meglio metterla in evidenza, ho costruito la curva della Tavola seguente, nella quale sulle ordinate sono segnati $i$ giorni di incubazione e sulle ascisse la perdita in peso in grammi riferita a 100.

Tavola V.

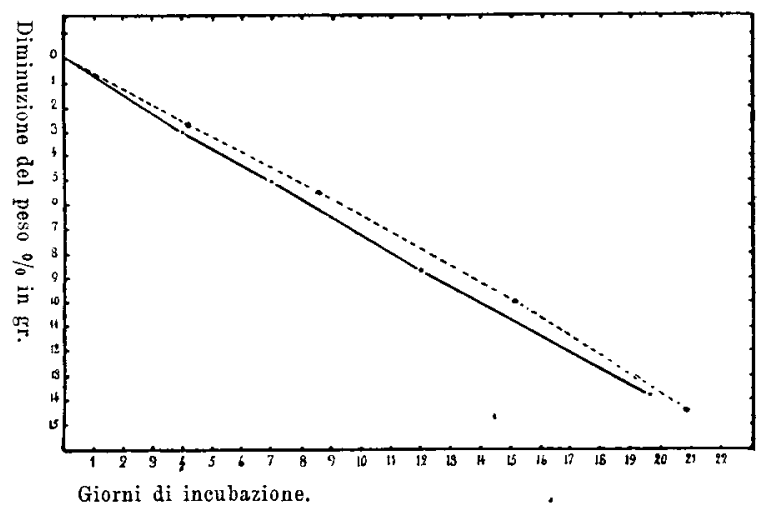

La curva superiore punteggiata corrisponde ai valori medi $\%$ dell'esperienza 25 agosto 1911, fatta al Colle d'Olen. La curva inferiore corrisponde ai valori medi \% dell' esperienza fatta a Torino, 20 aprile 1912. Queste due esperienze sono le più complete. La perdita in peso delle ova durante l'incubazione nell' aria rarefatta è più forte che al piano, specialmente nella prima settimana; le due curve in questo primo periodo sono nettamente divergenti. Nel secondo periodo dell'incubazione, la perdita in peso giornaliera nelle due serie d'esperienze diventa quasi di eguale intensità e le curve hanno la tendenza di farsi parallele. Alla fine della seconda settimana di incubazione, le ova nell'aria rarefatta hanno perduto circa $1.1: 100$ in peso più delle ova rimaste alla pressione normale.

Nell' esperienza fatta a Torino, si osserva che alla fine dell'incubazione la perdita in peso giornaliera delle ova non nate è molto forte, ma poichè le ova in quest'ultima parte dell'esperienza erano 
poche, non possiamo dare gran valore alle ultime pesate. Tuttavia è probabile che la perdita in peso anche delle ova nate a Torino, negli ultimissimi giorni debba aumentare per l'iniziarsi della respirazione polmonare, ciò che non può avvenire nelle ova incubate al Colle d'Olen, nelle quali lo sviluppo del pulcino non arrivò mai a termine.

La differenza fra la perdita in peso delle singole ova è talora fortissima, sia nelle esperienza fatte a Torino che in quelle fatte al Colle d'Olen, tuttavia si osserva che, per ogni ovo, la perdita in peso giornaliera rimane costante, forse perchè la maggiore e minore perdita in peso dipende dalla speciale struttura fisica e chimica del guscio delle diverse ova. Anche Port e Preyer avevano osservato che dal $1^{\circ}$ giorno di incubazione alla $2^{a}$ metà della $3^{a}$ settimana la perdita in peso è una funzione lineare quasi perfetta per ogni singolo ovo, anche se l'embrione era morto durante l'incubazione.

Il peso dell'ovo e la sua superficie non hanno un'azione evidente sulla maggiore o minore perdita d'acqua e nemmeno l'età delle ova; alcune ova messe nell' incubatrice parecchie stettimane dopo che erano state deposte, quando il loro peso era certamente diminuito in modo considerevole, mostrarono durante l'incubazione una perdita in peso non minore delle ova fresche. Non essendo possibile confrontare le pesate fatte sulle singole ora in montagna e al piano per le forti differenze individuali, per àvere un termine di confronto, è necessario eseguire esperienze sopra un numero grande di ova e valutare le medie.

Come abbiamo detto, lo sviluppo delle ova al Colle d'Olen non procedeva regolarmente e gli embrioni generalmente morivano al principio della seconda settimana; non ho potuto ancora stabilire quali sieno le vere cause di questo arresto di sviluppo. É noto che la disidratazione rallenta i fenomeni vitali: Bataillon ${ }^{1}$ ) con esperienze sulle ova di Ascaris ha confermato questo fatto, già sostenuto da GIARD ${ }^{2}$, ma nel nostro caso non eredo che la disidratazione maggiore che subiscono le ova nell' aria rarefatta possa essere la causa della morte del pulcino, giacchè questa non avviene prima in quelle ora che perdono maggior peso. Dobbiamo tenere presente che nell'aria rarefatta le ova durante l'incubazione possono subire più profonde

1) M. E. Bataillon, La pression osmotique et l'anhydrobiose. Compt. Rend. Soc. Biol. Vol. 52. 1900. p. 437.

2) Giard, L'anbydrobiose ou ralentissement des phénomènes vitaux sous l'influence de la déshydratation progressive. Compt. Rend. Soc. Biol. Vol. 46. 1894. p. 497. 
alterazioni per la diminuita tensione parziale dell'ossigeno e dell'anidride carbonica dell' ambiente. Lo sviluppo dell'embrione pare infatti influenzato dalla tensione dei gas che esso respira: BaUdRIMonT e M. SANT-ANGES ${ }^{1}$, e POT' ${ }^{2}$ ), hanno studiato l'azione dell'aumentata tensione dell'ossigeno, mettendo ova in via di sviluppo per parecchie ore nell'aria sovrossigenata; essi riscontrarono alterazioni evidenti. Più recentemente HASSELBALCH ${ }^{3}$ ) ha trovato che l'aria sovrossigenata, non aveva sempre la stessa azione sulle ova in via di sviluppo, talora dava un aumento, talora una diminuzione degli scambi respiratorii e talora anche restava senza effetto, forse perchè non esisteva una resistenza individuale costante contro l'ossigeno. In oltre, dobbiamo ricordare che il viaggio lungo fatto dalle ova per arrivare sino al Colle d'Olen poteva avere diminuito la loro resistenza: nelle ova rispedite al piano come controllo, si osservò appunto una minore percentuole nelle nascite.

Nella prima parte di questa Nota, abbiamo veduto che l'nomo e gli animali adulti in alta montagna eliminano una minor quantità di acqua, sebbene le condizioni fisiche dell' ambiente facilitino l'evaporazione dell'acqua dalla pelle e dalle mucose: il pulcino in via di sviluppo si comporta diversamente ed ubbidendo alle leggi fisiche, elimina in alta montagna una minor quantità di acqua che al piano. Però bisogna osservare che le condizioni d'esperimento non sono le stesse quando si studia la perdita d'acqua dell' ovo e quella dell' nomo $o$ degli animali al piano $\mathrm{e}$ in montagna. Nell'incubazione in alta montagna le ova rimangono alla stessa temperatura, solo diminuisce intorno ad esse la tensione parziale del vapor d'acqua pur continuando ad esserne satura l'aria dell' incubatrice, ma per l'uomo e per gli animali si modifica anche la temperatura, che diminuisce. Tuttavia, pare dalle esperienze citate di GiLlemard e Moog che, anche rimanendo costante la temperatura, l'animale adulto nell'aria rarefatta elimini meno acqua che alla pressione normale, perciò esso possederebbe realmente certi poteri di regolare l'evaporazione, che non possiede l'animale in via di sviluppo.

1) Baudrimont et M. St. Anges, loc. cit. p. 235-238.

2) R. POTT, Versuche ïber die Respiration des Hühner-Embryo in einer Sauerstoffatmosphäre. PrLügers Arch. Bd. 31. 1883. S. 268.

3) Hasselbalch, loc. cit. S. 395 . 
648 Alberto Aggazzotti, Influenza dell' aria rarefatta sull' ontogenesi. I.

\section{Conclusioni.}

$1^{\circ}$. La perdita in peso dell'ovo durante l'incubazione per l'evaporazione dell'acqua è maggiore in alta montagna che al piano.

$2^{2}$. L'embrione di pollo nell'incubatrice, durante lo sviluppo, non si comporta come l'animale adulto, che in alta montagna ha una minor perdita d'acqua che al piano.

3. L'embrione di pollo, a differenza dell'animale adulto ubbidisce alle leggi fisiche, che in alta montagna richiedono una evaporazione maggiore che al piano; esso non possiede adunque nessun potere regolatore alle nuove condizioni, come possiede l'animale adulto; forse in causa della più bassa temperatura.

\section{Zusammenfassung,}

1) Der Gewichtsverlust der Hühnereier durch Ausdünstung des Wassers während der Bebrütung ist im Hochgebirge größer als in der Ebene.

2) Der sich im Brütkasten befindende Hühnerembryo verhält sich während der Entwicklang nicht wie das erwachsene, frei lebende Tier, das im Hochgebirge einen niedrigeren Wasserverlust als in der Ebene erfährt.

3) Der Hühnerembryo folgt also im Gegensatz zum Erwachsenen den physikalischen Gesetzen, die im Hochgebirge eine größere Ausdünstung als in der Ebene verlangen; er weist also bei den gegebenen Verhältnissen keine Re. gulationseinrichtung fiir den Wasserverlust auf, die bei dem Erwachsenen vorhanden ist, jedoch mit den Temperaturverbältnissen zusammenhängen kann. 